\title{
Can We Quarantine the Quantum Blight?
}

\section{Craig Callender}

The science fiction novel Quarantine portrays a world wherein interaction with human observers is necessary to collapse quantum wavefunctions. The author, Greg Egan, amusingly puts the emphasis on the observers being human - aliens can't do it. Aliens are therefore at a tremendous disadvantage. As we gaze at the night sky, we are constantly collapsing alien worlds, depriving them of their branch diversity. Whole civilizations are being snuffed out by our observations! Understandably the aliens grow tired of this. In response they erect an impenetrable shield around the solar system, one that blinds us to the outside universe. This shield protects the rest of the universe from harmful human observation, locking humanity into a starless Bubble.

When confronting scientific realism with quantum mechanics, many philosophers advocate the theoretical counterpart of this fictional strategy. Quantum mechanics is beset with notoriously difficult interpretational challenges. Differ-

ent interpretations of the theory are compatible with present data. Only the most unreconstructed positivist thinks these different interpretations (different theories, really) are notational variants, i.e., different representations of the same facts. Scientific realism holds that most of the statements of our mature scientific theories are approximately true; but this claim is threatened by persistent underdetermination of theory by evidence, for one theory isn't better confirmed than its rivals. Faced with this threat, some try to lock the quantum interpretation problem into a theoretical Bubble, cordoning off the interpretational blight and leaving the rest of the world safe for scientific realism.

My goal in this paper is to pop this Bubble. No shield can really protect the poor aliens in Egan's story, nor can any theoretical membrane protect scientific realism from dealing with the quantum measurement problem. One may be able to erect barriers around the observable or classical, preserving a realism about tables, chairs and the like, but there is no safety zone within the quantum realm, the domain of our best physical theory. The upshot is not necessarily that scientific realism is in trouble. That conclusion demands further arguments. The lesson instead may be that scientific realists ought to stake their case on particular interpretations of quantum theory. In any case, the realist can't ignore the interpretational issues plaguing quantum mechanics. 


\section{The Quantum Blight}

Quantum theory is one of the most successful sciences we have ever developed. It is a rigorous formalism attached to rich experimental practices. Together, the formalism and experimental practices allow us to make bold and novel predictions that have been confirmed time and again for over 90 years. Unfortunately, it's not clear what world is being described by this theory. We need to know the "word-world" connections. What do the terms in the formalism represent in the outside world, if they represent? For example, does the wavefunction represent our knowledge, a real field evolving in a high dimensional space, a complicated field on a low-dimensional space, an aspect of a law of nature, or what? The question isn't solely directed at the quantum state. It applies to everything in the theory - the $q$ 's, $p$ 's, $\sigma$ 's, and more.

Word-world questions arise with every theory. Does classical particle mechanics portray a world with forces, with three equal types of mass (active, passive, inertial) or one type? Options exist. The main difference with the situation in quantum theory is that quantum theory, unlike classical mechanics, suffers from the infamous measurement problem. The measurement problem in effect shows that the word-world connections offered by the standard "Copenhagen" interpretation are inconsistent - or at the very least, lack theoretical virtues that we normally expect of a theory (see, e.g., Bell 1987). It's a huge flag calling attention to the need for clear and consistent representational connections for quantum mechanics.

Answers exist. Too many. There are Bohmians, followers of Bohm 1952, who hold that quantum mechanics is incomplete and supplement it with additional ontology. There are advocates of Collapse, like GRW 1986, who propose modifications to the linear wave evolution. There are Everettians who posit a kind of multiverse (Everett 1957). Hybrids of all three theories exist. For instance, one can divide Collapse interpretations into "Everettian Collapse" and "Bohmian Collapse" theories, depending on whether the theory posits beables in addition to the wavefunction (Allori et al 2008). A similar claim can be made for Everett, as one can interpret Everett as positing a matter density distribution like GRW (Allori et al 2011) or even create a kind of Bohmian multiverse (Sebens 2015a). Answers with a more "pragmatic" or "instrumentalist" flavor exist, including Healey's recent pragmatic view and Fuchs' Quantum Bayesianism (see Healey 2017 and this volume for discussion and references). The diversity of worlds possibly described by quantum mechanics is shocking. One might stubbornly insist that the difference with classical mechanics is one of degree, that both theories have unresolved word-world questions. Fine, but the number of degrees is huge. Nothing compares classically, for example, to the contrast between the sparse ontology of GRW (with "flash" ontology) and the generous ontology of Everett.

The interpretations describe dramatically different ontologies, but more than that, they typically offer different laws of nature and different core theoretical edifices. Collapse theorists modify the linear dynamical evolution of the wavefunction. Bohmians offer a guidance equation for their particles or fields. It's 
hard to find a theoretical core that they all have in common such that we can regard them all as different interpretations of that core. Even the operator algebra that is taught in every quantum text is contested: for instance, for Bohmians, Hilbert space and the operator algebra are an emergent measurement formalism having no place in the fundamental description of nature, whereas for Quantum Bayesianism, that formalism is the core. For these reasons the many "interpretations" are clearly different theories.

A clearer picture is painted by conceiving these "interpretations" as different Lakatosian research programs (Lakatos 1978). A research program is a series of theories sharing a "hard core" of temporarily unimpeachable theoretical posits. Quantum mechanics is a live theory, one being extended to new forms and realms. The non-relativistic theory of 1925 is applied to new systems daily, from ever more sophisticated treatments of helium to the recent discovery of non-equilibrium time crystals. The theoretical structure was also transformed into QED, QCD and the standard model, and we hope to integrate cosmology and gravity with the quantum. The interpretations typically have something to say about all these developments. Often how they respond changes the laws and ontology posited by the theory, e.g., as we'll see, Bohmian quantum field theory may posit different ontology than non-relativistic Bohm theory. With so much different, in what sense can we speak of an interpretation or theory? The answer is that each "interpretation" is really a research program.

Lakatos' "negative heuristic" is that which is unrevisable in each program and defines its "hard core": Everettians all hold that macroscopic superposition indicates multiplicity; Bohmians all postulate ontology guided by a new equation hooked up to a wave equation; advocates of Collapse all modify the wave equation to produce a unified story of the macro and micro realms; Quantum Bayesians are committed to the idea that wavefunctions represent the amount of information one has about a system. These hard cores are carried along when each "interpretation" is applied to some new domain or theory. Lakatos' "positive heuristic", by contrast, is that which is revisable within each program. Bohmians can propose new choices of ontology to be guided by the wavefunction if they are better suited to the pheneomena, Collapse theorists can tinker with the size, timing and triggers of collapse. Thus understood, even dramatic departures, such as Collapse approaches to semi-classical quantum gravity (Okon and Sudarsky 2015) and Bohmian approaches to superstring theory (Weingard 1996), are easily recognized as descendants of the original families.

Carve answers to the measurement problem into four broad research programs. Little hangs on this division, and I'm happy to acknowledge that different partitions and hybrid theories exist. Using one reasonable partition, we find four active research programs: Bohmian, Everettian, Collapse, and Pragmatist/Bayesian programs - each very broadly construed. The last of these programs doesn't aspire to characterize or represent a complete physical reality. These views are often accused of being instrumentalist interpretations. Whether this accusation is fair or not, this last set of programs will not be relevant to realism and the present worry of underdetermination because it doesn't offer us a representation of physical reality. We therefore have three live research pro- 
grams (Bohm, Collapse, and Everett), each portraying dramatically different realities (for accessible discussions of each, see Maudlin forthcoming).

Confined to nonrelativistic quantum mechanics, experimentally there is no way to confirm one over the other. Bohm and Everett use precisely the same algorithm for extracting predictions (Born's rule). Collapse typically uses a slightly modified one that differs negligibly in the macroscopic realm from what Bohm and Everett use. Philosophers sometimes raise the distinction between pairs of theory being "in principle" undermined by data and other pairs merely being "in practice" so underdetermined. Collapse and Bohm/Everett, they might say, are then in principle empirically distinguishable unlike Bohm and Everett. But as we know from Laudan and Leplin 1991, it's not clear that the "in principle versus in practice" distinction is itself an in-principle one. And when comparing research programs as opposed to artificially frozen theories and fixed empirical domains, it's not clear that this distinction is so useful. There are plenty of experiments we can imagine that would provide a crucial test of Collapse against other theories. Collapse posits two new constants of nature, the collapse width and the collapse rate. Some choices of these parameter pairs have already been "falsified" by experiments involving spontaneous x-ray emission. Had the original GRW theory chosen such a pair, the theory would now be demonstrably false. However, with room remaining in the "unfalsified" parameter space, an advocate of Collapse in that scenario could simply shift to a new parameter pair, saying that he or she learned better. The Collapse research program can survive falsification of some particular parameter pairs. For this kind of reason, no Popperian crucial test between research programs is likely in the foreseeable near future. ${ }^{1}$

\section{Dialing Up Underdetermination}

Underdetermination of theory by data is a phenomenon that can happen, as the phrase suggests, when the empirical data do not narrow down the space of acceptable theories to one. As a logical matter, left at this, this situation is guaranteed to always obtain. We know from the curve fitting problem that a finite number of data points can be connected via an infinity of curves. If we treat each curve as a theory, then we always face massive underdetermination of theory by data. In the philosophy of science, however, we curiously restrict the available theories to properly "scientific" ones. I say this is curious because philosophers of science, of all people, know that the label "scientific" is notoriously vague. What is meant?

\footnotetext{
${ }^{1}$ Detractors of each program may object to what I've said here. Critics of Everett will insist that Everettians cannot recover the Born rule predictions due to the theory's well-known problem with probabilities, so we don't know if it's empirically adequate. Critics of Collapse will raise worries about the tails problem and related threats to the available parameter space (Sebens 2015b). Critics of Bohm will point out that it hasn't been fully extended to quantum field theory and is therefore not empirically adequate in the relativistic realm. Each worry is very serious. I explicitly tackle the last in section 5 . However, for present purposes, because I'm discussing programs as opposed to static theories, I'm inclined to be generous and hope that each can overcome their challenges, especially the ones they're actively working on.
} 


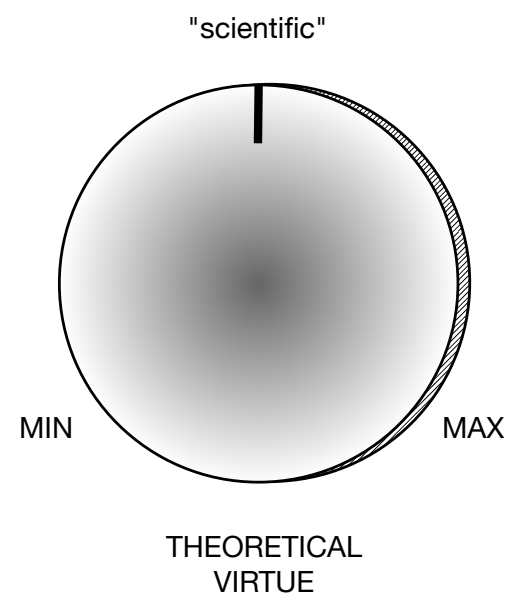

Fig. 1: The Scientific Dial

Imagine a dial (see 1) that we can set to more or less "scientific." Admittedly oversimplistic, we might think of the settings as measuring increasing theoretical virtues. The lowest setting might be mere logical consistency. When the dial is set there all sorts of wild and intuitively "unscientific" theories count. Skeptical nightmares like Descartes' demon theory and Putnam's brain-in-a-vat theory count as scientific because they are consistent. In terms of curve-fitting, this setting allows even the most "wiggly" of curves. The result of this theory is massive underdetermination. Turning the dial up winnows down the number of contending research programs. Suppose we insist not only on consistency but also on the theory being predictively useful to human beings, unified, consilient, simple, etc. Then when we turn the knob Descartes' demon theory drops out because it isn't predictively useful to human beings. In principle, being super strict with what we mean by "scientific" could winnow the acceptable theories down to one. Let's not go that far. The philosopher of science dealing with underdetermination instead sets the dial at a sense of scientific that a consensus would agree upon as genuinely scientific, a sense that would separate good scientific theories from pseudoscientific claims (e.g., evolution from creationism). What happens?

Arguably, at a sufficiently coarse-grained level, such a setting in biology restricts the available theories down to one, namely, the modern synthesis of molecular biology and Darwinian selection. No serious scientific rivals exist, although of course at a finer scale all manner of controversy erupts. By contrast, 
if we focus on quantum phenomena, we are left with our four programs, three of which describe in detail very different worlds. Although many of the terms used are a bit vague, still the contrast between the situation in quantum mechnaics and modern biology is striking.

The three quantum research programs pose a prima facie threat to scientific realism. The realist holds that most claims about observable and unobservable facts made by a mature scientific theory are true or approximately true. Agree for the moment that Collapse is mature and successful. One can't defend a belief in collapses if one at the same time admits that the evidence equally well supports a theory without them.

Are we really in this situation? Prima facie, yes. In a perceptive paper John Norton 2008 attacks the idea that underdetermination is guaranteed. The artificial playthings of philosophers that serve to justify a state of permanent general underdetermination fail, he thinks. I agree. There is no automatic proof of general underdetermination. It's not easy meeting the demands of the above dial when it is set high. However, Norton also hints that most cases, general or not, are probably not threatening. Is that the case here? Consider two theories underdetermined by the data, $T$ and $T^{\prime}$. Let's examine the three cases he envisions.

First case: $T$ and $T^{\prime}$ predict the same observational evidence $E$ and they are inter-translatable about unobservable content. That is certainly not the case for our three programs. There are no translations of a Collapse swerve nor a Bohm particle into their rivals, respectively.

Second case: $T^{\prime}$ is parasitic upon $T$, but $T^{\prime}$ is epistemically inequivalent to the original. Suppose $T$ implies $E$ and that $T^{\prime}=T \& H$. Then as Norton points out, good theories of confirmation do not automatically agree that if $E$ confirms $T$ it also confirms $T^{\prime}=T \& H$, where $H$ is some arbitrary hypothesis. Again, none of our three research programs are parasitic upon one another in this sense. One encounters the claim that Bohm is Everett "in denial". The thought is that Bohm simply adds a hypothesis about which Everett "branch" is occupied by particles. However no one would claim that this is parasitism in this cheap "\&H" sense. If it were, it would be trivial to generate successful field extensions of Bohm - but it's not. So if parasitic, it's not in this automatic sense. In any case, the "in denial" charge is in my opinion wrong for many reasons and on some interpretations not remotely plausible (Callender 2015 and references therein).

Third case: $T$ and $T$ ' are not inter-translatable but "similar," and Norton thinks, therefore likely to be theoretically identical. Norton doesn't say why similarity makes identity likely, but let's grant him that it does raise the suspicion. He suggests that Bohm and Copenhagen are in this relationship. It's hard to understand the reason why, as Copenhagen isn't consistent, or if it is, it seems to fundamentally cleave the world into classical and quantum regimes according to fuzzy rules - neither of which is the case in Bohm. Nor are the theories similar structurally, as the Hilbert space formalism that is central to Copenhagen isn't a crucial part of Bohmian mechanics. Unless similarity is understood as simply empirical equivalence, there is little reason to take the three 
research programs considered here to collapse into one.

With these research programs, it seems that we face the realist's nightmare. Many philosophers suspected that the threat of underdetermination is artificial, confined to excessive flights of imagination and not genuinely scientific theories. However, these three programs are neither philosopher's toys nor notational variants (on any remotely reasonable semantics) and are clearly "scientific" in letter and spirit. Quantum underdetermination is the real deal.

\section{Underdetermination within Underdetermination?}

Quantum underdetermination may be worse than just characterized. I described three programs, but there is potentially a lot of underdetermination within each too. My hope, however, is that many of these empirically equivalent theories will turn out to be uncontroversially epistemically inequivalent. The normal process of scientific discovery will weed them out. One might say the same about the underdetermination we just confronted - i.e., hope that it goes away - but what I have in mind at present are relatively uncontroversial choices dictated by theory development.

Consider Bohmian mechanics. Central to the theory is the choice of a beable (e.g., particles) and a guidance equation describing the dynamics of that beable. There is some freedom in both choices. Let's take a look.

Bohmians provide a dynamics for their beables to "surf" wavefunctions. Wavefunctions evolve according to linear wave equations in quantum mechanics, such as the famous Schrödinger equation. Bohm's theory relies on a crucial feature of such equations, namely, that they imply a continuity equation, a local form of conservation. What is conserved is the probability density through time. That density determines the chance of finding a particle at a location at a time. Bohmian mechanics is based on the simple insight of using this conservation and its associated conserved current to define the velocity guiding the beables, just as one does in fluid mechanics and elsewhere. Supplemented with the claim that the particles are initially randomly distributed, the theory is empirically adequate in the non-relativistic regime.

Many other choices of guidance equation also prove to be empirical adequate. Add any divergence-less vector field (divided by the probability density) to the original velocity. The continuity equation does not "see" this addition. Hence this new modified velocity will also be empirically adequate. Yet this additional vector field is arbitrary, characterizing indefinitely large and potentially wild deviations from the original Bohm velocity.

This case may be a benign form of underdetermination. When discovering scientific theories, scientists use a variety of non-empirical issues as guides. Some, like simplicity, raise worries because simplicity may be in the eye of the beholder, but other considerations seem uncontroversially "scientific" and are a

poor basis for serious worries about underdetermination. Such considerations may constrain the form of the Bohm velocity. Durr et al 1992, for instance, claim that the original choice is the unique Galilean-invariant velocity (but see Skow 
2010). Peruzzi and Rimini 2000 claim that it is the unique choice that works also for the center-of-mass of the Bohmian configuration, a desirable feature for many reasons. So there is plenty of reason to expect the form of the velocity to be whittled down by ordinary scientific reasons encountered in discovery.

Turn to the choice of beable. Consider two cases, one, the choice in the original theory, and two, the choice when we move to field extensions. The usual choice in the non-relativistic particle theory is to choose particles with determinate position as the basic ontology. There are good reasons for this choice, as other choices such as momentum don't solve the measurement problem. However, it's well-known that one can add additional beables to the theory, such as spin. In fact, there is a general recipe for adding new "basic" properties to Bohm particles (see Holland 1995). These additions do seem akin to Norton's parasites. The measurement outcomes are recorded in position ("up", "down" and so on). The wavefunction and particle positions together entail the spin vector representing the spin beables. Absent an independent reason to exploit the spin vector, it seems that Occam's razor will quickly remove the basic spins from the Bohmian particles — and with them this alternative formulation.

Turning to quantum field theory, matters really open up (see Struyve 2011 for an excellent review). Bohmians face choices between adding particle or field beables. In his original paper, Bohm proposed a field beable for the electromagnetic field, an actual field configuration corresponding to the transverse part of the vector potential. One can do something similar for other boson fields, but this approach is hard to extend to fermionic fields. A radical response to this trouble is just to get rid of fermions altogether. Measurement results will get recorded in bosons, so in some sense they are "enough" for empirical adequacy. On this "minimalist" approach, there are no fermions but the wavefunctional carries a label representing what would be their degrees of freedom - so the boson field behaves as if there were fermions around. One can dress up the bosonic fields via the method used for spin vectors mentioned above, providing a sense in which there are fermions, but Occam's Razor will slice these properties away as quickly as it would the above spin properties. The other way to go is with particles rather than fields. Ironically, the particle picture works well for fermions but less well for bosons. In Bell 1987 fermion particle number is defined but there is no configuration for bosons. We can also entertain a hybrid theory, one treating fermions as particles and bosons as fields, which is how we treat electrons and photons in classical electromagnetism. In sum, we have choices between particles or fields and even whether bosons or fermions exist! Then again, the overdetermination here may be overstated. Right now approaches are getting eliminated or favored for normal reasons of physics, e.g., no natural measure for Grassman fields, Euler angles not solving the measurement problem. Work is ongoing, and as theories are extended they meet more constraints. It would be premature to say that quantum field theory yields rampant underdetermination for Bohmians.

I've concentrated on the Bohmian case, but the other two research programs face similar issues. If Collapse posits non-wavefunction beables (e.g., matter density, flashes), one will face similar questions about what is the right beable. 
There are also additional choices: the hit rate and collapse width, the "trigger" for collapse (particle number, mass, Weyl curvature, Riemannian curvature), and more. Everettian theories likewise need to choose whether to add a beable (e.g., mass density) or not. Even if not, questions remain that can possibly lead to numerous theories, such as determining the microscopic ontology of the Everettian world. Wallace and Timpson 2010 make one proposal (spacetime points with properties) but there are alternatives. As in the Bohm case, I suspect that most of these decisions will be decided by normal theory development and not cause widespread underdetermination. That said, given the uncertainty, we enjoy no guarantee that this will be the case.

\section{Quarantine}

If what I've argued is on the right track, then we have serious scientific underdetermination of theory by data striking right at the heart of our most basic successful scientific theory of the world. One natural reaction is to quarantine this underdetermination to some specific regime and free some theoretical claims from epistemic danger. The intuition behind the quarantine strategy is that the disagreement between these camps is isolated to esoteric bits of the theory. These esoteric bits are where physicists have little confidence; instead, what they are confident about are the core explanations of typical quantum phenomena, and on these, each camp agree. Where the three camps agree on some theoretical claim, that claim is not subject to underdetermination. On its face this position strikes me as tempting and plausible.

Let's spell the position out slightly more carefully. To be interesting, the claims that the quarantine strategy must protect are

1. theoretical

2. not merely mathematical

3. specifically quantum.

Let me explain. Demand 1 should be uncontroversial. We're focusing on attempts to rescue realism. We already know that all three programs get the observables right - that's what it is to solve the measurement problem. Demand 2 should also be uncontroversial. We're after scientific realism, not mathematical realism. All the research programs agree that $2+2=4$. Yet that doesn't tell us much about unobservable contingent physics. Demand 3 is imposed because we're interested in whether realism can reach into the quantum realm. Just as the three programs may agree on the observables associated with macroscopic objects, they may also agree on some unobservables for some systems as we move into the classical limit. Quantum decoherence is a process whereby interactions among constituents of a system and its environment lead to the suppression of quantum interference. This process occurs in all three programs. The issue is tricky, but arguably after decoherence all three programs will agree on much. 


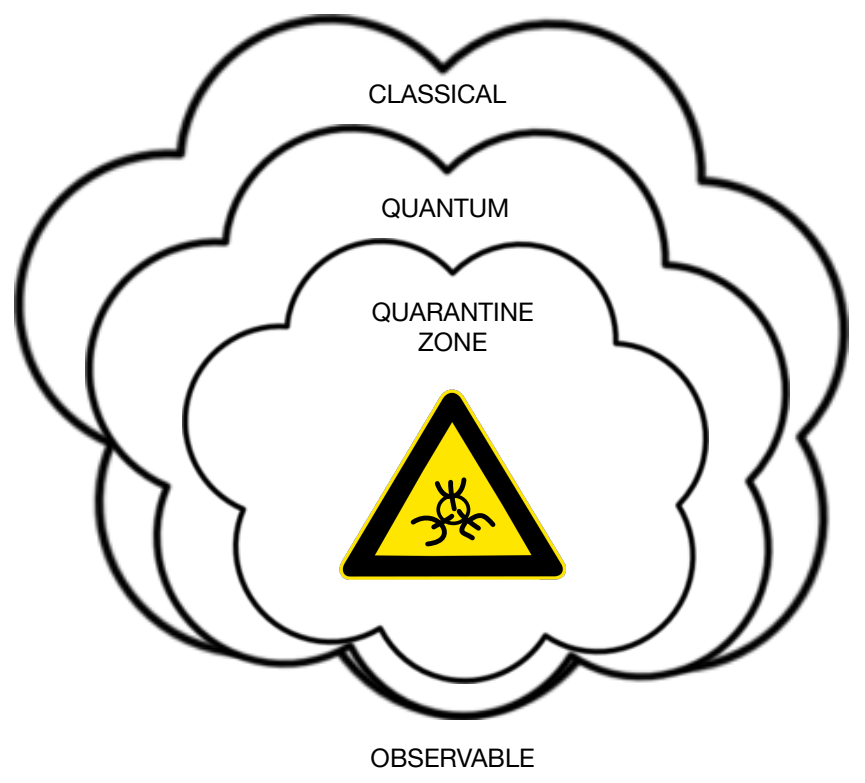

Fig. 2: Quarantine Zone

But that's not so satisfying if we want to know whether we should be realists about coherent quantum systems. Decoherence may provide a defensible quarantine strategy that frees some claims about unobservables, but hopefully we can do better than secure realism only in the classical domain of quantum theories.

The rules are set. Are there substantive claims about the specifically quantum realm that are shared by all three of our research programs? We know there is massive agreement from above: they all agree on the observables, perhaps even the classical limits. They all disagree way down below: for instance, we won't find continuous Bohmian particle trajectories in Collapse or Everett. What about in between? Although the real world and real theory of it are way more complicated, Figure 2 provides a toy model of the setup.

\section{1 “Textbook" Quarantine Zone}

In a little discussed paper, Alberto Cordero 2001 offers what I think is one of the best ways of finding a quarantine zone. His idea, as above, is that the three camps overlap considerably. In his own words:

the underdetermination at hand is clearly of limited scope...all the 
mentioned competitors associate the quantum state with a peculiar physical field, all include the Schrödinger equation centrally in the dynamics, all endorse a strong form of ontic-structural nonseparability, and all agree on geometrical relations between subsystems (internal molecular shapes, atomic and quark structure, etc.). By contrast, divergence between the competing models is peculiarly confined to certain specific respects and degrees of precision, with clear significance limited to some fundamental questions...So, although the case makes for an intense ontological debate, its corrosive power on belief seems confined to just some aspects of the full narrative. The encountered underdetermination does strike realist theorizing from a certain depth down, but then again only along certain lines of inquiry. 307

Having provided examples of convergence amongst our three programs, Cordero concludes that many hypotheses about the quantum world are safe from the quantum blight.

Cordero mentions many areas where the programs converge, but I want to focus on what I'll dub textbook quantum mechanics. By this I mean the narratives found in quantum textbooks about what's going on in quantum systems. Here I'm thinking about Cordero's claim that the research programs agree on "geometrical relations between subsystems (internal molecular shapes, atomic and quark structure, etc.)" but also similar examples he gives throughout, e.g.,:

Take, for example, the basic quantum mechanical model of the water molecule, with its atom of oxygen bonded to two atoms of hydrogen, the latter making with the former an angle of about $103^{*}$ in "normal" thermodynamical conditions. Stuff like this is contained in approximate partial models shared by all the viewed theories. . . . $[\mathrm{M}]$ uch in the quantum mechanical story about water molecules and their interaction seems at least as credible as the most ordinary talk about, say, cats and common objects. 309

Cordero doesn't associate his position with textbooks, so he shouldn't be saddled with my interpretation. If preferable, we can say this idea is inspired by Cordero's examples and not his position. In any case, I want to focus on this claim because it strikes me as new and interesting. Textbook physics seems interpretation neutral. We feel that we can trust what they say about typical quantum systems (e.g., claims about orbits, molecular structure, the behavior of energy, tunneling phenomena, and so on) while bracketting the measurement problem. At a certain "depth" trust runs out, e.g., whether the theory is deterministic or based on particles. Yet these are deep metaphysical questions that do not touch quantum textbook claims.

In fact, I think the conjecture about what I'm calling textbook quantum mechanics is more plausible than his initial claims quoted above. Tease apart what I'm calling the textbook claim from his initial claim that "all the mentioned competitors associate the quantum state with a peculiar physical field" and so 
on. That initial claim about the quantum state might strike readers as true-but at best it's only approximately true. The normalization one does in Collapse will slightly change the state used, and differences in decoherence might imply slightly different Bohmian effective wavefunctions from the wavefunctions associated with branches in Everett (the analogues of the quantum state assigned to sub-systems in each program). More importantly, the agreement may only exist at the mathematical level. What the quantum state represents can vary dramatically amongst research programs. Similar claims can be made about the dynamical equation, although here the differences between Collapse and the rest are starker. The most mathematically sophisticated versions of Collapse, continuous spontaneous localization theories, propose stochastic nonlinear wave equations; the stochastic modifications are crucial to the theory and have huge structural ramifications (e.g., regarding norm preservation). Structurally they are importantly distinct from the Schrödinger equation. For these reasons I want to reject Cordero's first set of claims.

Proponents of structural realism (McKenzie 2017) may insist that all three programs share substantial core structure, namely, the structure of Hilbert space, the operator formalism, commutation relations, Born's rule, and more. Structural realists modify realism by retreating to the mathematical or structural relations in a theory. But the claim of a common structure here would be overblown. The operator formalism - for all three programs — is simply a measurement formalism, a tool added to the core theory, not the theory itself. For the Bohmian, for instance, experiments define maps from initial wavefunctions to distributions of particle positions. Bohmian commitments imply this map is bilinear. Bilinear maps are equivalent to positive operator valued measures and the traditional quantum operators are particularly simple expressions of these (Daumer et al 1996). Bohmians could in principle just speak of the particle distributions directly and skip all of this - at least as far as fundamental theory goes. The same goes for the Everettian but regarding the quantum state. The conventional "word-world" connection used by Copenhagen is abandoned. That interpretation understood the operator formalism as being a guide to the "properties" of a system (a system has a property associated with an operator iff its state lives in the subspace that the operator projects the state onto). But none of the programs we're looking at employ this connection. Stripped of its connection to the unobservable quantum world, what's left behind is a useful algorithm for predicting measurement outcomes and no more. True, at some emergent measurement level that algorithm is shared. Yet that level is observable and therefore not relevant to rescuing any kind of realism. The operator formalism is the wrong place to look for substantial physical overlap.

Back to quantum textbooks. Cordero's claim, disentangled from structural realism, is independently attractive. When we probe it, however, it faces trouble. Recall that for realism to be interesting in this context, theoretical claims must satisfy at least three demands. They must be non-mathematical, theoretical, and specifically quantum. Do Cordero's examples meet these criteria? Do other quantum textbook claims? I think it's pretty clear that these claims are not held in common amongst programs, not with each other, and not with what 
textbooks say. Divergent physical pictures emerge as soon as we peek into anything quantum. Extracting "what textbooks say" can be a bit of an art, but I suspect my readings agree with conventional wisdom. I'll typically focus on the Bohm case, as it provides many worked out physical systems that diverge sharply from the quantum textbooks, and crucially, the other interpretations; but this focus is for convenience only.

\subsection{Water and Bonds}

Let's begin with the assertion quoted above about water, its bonds, internal angles, and so on. These propositions are non-mathematical and about the unobservable level, but are they quantum? Not necessarily. Claims about the composition of water, derived from stoichiometry, go all the way back to Lavoisier! Crystallography and x-ray diffraction then added to our knowledge of water's structure, but this was based on theoretical work by G.N. Lewis and experimental work by van Laue - both safely pre-quantum. The currently used angle of 104.5 degrees is based on crystallography experiments. Cordero may respond that the experimental value is often considered a confirmation of quantum effects, as a simple textbook quantum treatment puts the value at the nearby 109 degrees. But we could still say what Cordero does about water had neither Schrödinger nor Heisenberg ever existed. Experiment plus some minimal non-quantum theory would have been enough.

The danger of confusing insights from experiments for quantum posits exists even with water's subcomponents. Peeking at hydrogen (more in a moment), note that the accepted bond strengths and bond lengths are based entirely on classical physics and experiment. Other claims about orbits, deriving from the famous Bohr model, are based upon semi-classical theories. Claims from such theories are not quantum. A fully quantum treatment of hydrogen will include specifically quantum effects. I grant that a vague boundary exists between what is quantum and what is not in chemistry. Linus Pauling's famous work on the chemical bond is probably the beginning of a fully quantum treatment, although I'm no historian and I'm happy to concede the boundary to Cordero.

In any case, our first lesson is that many plausibly "safe" statements about bonds and angles are not truly quantum.

\subsection{Tunneling}

Tunneling is without doubt a purely quantum effect, one studied in every quantum textbook. It was used by Gamow and Gurney and Condon to explain the emission of alpha particles from unstable nuclei. Because the attractive potential of the nuclei is much larger than the kinetic energy of the alpha particles, such observed emission is impossible classically but possible quantum mechanically.

Textbooks typically explain how tunneling is possible by treating a system of particles of energy $E$ incident from the left beamed at a one-dimensional potential step of height $V$, where $E<V$. An approximate plane wave solution to the Schrödinger equation is given, where the wavefunction to the left of the 
barrier is supposed to represent a superposition of a wave going to the right toward the barrier and a reflected wave going to the left, and the wavefunction to the right of the barrier represents a wave that is transmitted through the barrier. Textbooks calculate the probability of transmission by showing that it is a function of the incoming and reflected fluxes, demonstrating that for certain ratios this probability is non-zero - hence demonstrating the possibility of tunneling.

Given this wavefunction, one can work out the Bohmian trajectories that are then implied. Assuming it is a case of tunneling - and therefore that the incident wave is bigger than the reflected one - it follows that the probability current is positive. Because the probability density is positive, the velocity of the particles is therefore positive too. Hence there is nothing reflected at all. The alleged "particles that reflect" actually all have positive velocity toward the right! So there is no reflection even when the reflection coefficient is non-zero and the transmission coefficient is not one. The reflection/transmission coefficients used in the textbooks don't have anything to do with the actual Bohmian motion. Here we have a massive departure from the physics promoted by the textbooks. You might reasonably have hoped that all the interpretations would agree with the minimal implication found in the textbooks on the most canonical system, namely, that something is reflected to the left. That is not the case. ${ }^{2}$

\subsection{Hydrogen}

This case is simple but instructive. The hydrogen atom was the first system treated quantum mechanically and is a staple of every textbook. The electron is said to orbit the nucleus - or sometimes something vague about a "probability cloud" extending a certain distance from the nucleus's center is mentioned. But in Bohmian mechanics, as is well-known, stationary states such as $\psi=|1 s\rangle$ and $\psi=\mid 2 s>$ have constant phase and therefore the electron is at rest with respect to the nucleus. (In the quantum potential approach to Bohm, what happens is that the so-called "quantum potential" $Q$ balances the classical potential $V$, holding the electron a fixed distance from the nucleus. $Q$ thus provides the quantum "pressure" keeping the electron from crashing into the nucleus as predicted classically.) Here we have no orbits at all, contrary to the textbook picture!

This situation can happen even when the orbital angular momentum quantum number $(l)$ is greater than zero. One can have (a kind of) momentum without motion. The lesson is that "[q]uantum numbers do not directly represent dynamical properties" (Holland 1995, 156). This is an important point, as the textbooks mostly assume that quantum numbers do reflect properties of

\footnotetext{
${ }^{2}$ I admit that this example is an artifact of an inadequacy of the textbook treatment of tunneling. The normal textbook treatment via plane waves is flawed: it's not clear how such stationary states justify talk of entities moving from the left and so on; worse, these waves are not renormalizable, so they aren't physical. A better treatment is possible (see Norsen 2013). Nonetheless, this case is a nice one to use here because this (albeit flawed) textbook treatment of tunneling is so common and the straightforward Bohmian consequence is so at odds with it.
} 
a system. An advocate of the quarantine strategy might hope for that much. If we briefly turn to collapse theories, note that on a "flash" ontology picture a lone hydrogen atom is most likely literally nothing. The world is populated with flashes only when collapses occur, but we would in all likelihood have to wait thousands of years for a hydrogen atom to collapse. On a mass density interpretation, by contrast, the mass or matter will be smeared out across all components of the superposed atom.

Bohmians and textbooks don't agree about hydrogen until the observable level. Agreement exists because the observables require an experiment, and hence a physical interaction, one that changes the state of the hydrogen atom (and in particular, gets the electrons to where they need to be). Below the surface of the observable Bohmians also disagree with Collapse and Everett about the behavior of hydrogen atoms.

\subsection{Two Path Experiments}

Consider the sort of typical two-path interference experiment commonly found in textbooks. A spin-1/2 particle enters a Stern-Gerlach device oriented so as to separate $x$-up from $x$-down particles. Depending upon its initial position, the Bohm particle either follows the upward wavepacket or the downward one. Deflectors are added to the setup that deflect the upward wavepacket downward and the downward one upward. The wavepackets meet at location I, symmetric between the up and down paths, and then continue on their way, the initially upward wavepacket heading downward to A' and the initially downward wavepacket heading upward to B'. Measurements can be made at A' and B'.

Textbooks describing such a case of course do not assume that there are particles traveling definite trajectories. Often we're told that because the particle is in a superposition it simultaneously travels both paths. Whatever is going on, it is assumed that if the particle is measured at $A$ ' then it came from $A$ and if it is measured at $B$ ' then it came from $B$. Something is traveling from $A$ to $\mathrm{A}^{\prime}$ or B to B' or both.

What happens in Bohm's theory? Because the Bohm velocity equation is first order and deterministic, trajectories cannot cross in configuration space. That fact, coupled to the additional fact that spin is a feature of wavefunctions and not particles, force Bohmian trajectories sometimes to behave in highly nonclassical and surprising ways. In the experiment at hand, due to the symmetry of the setup, trajectories would have to cross at I for a particle from A to go to A' or from B to go to B'. Hence the probability of finding a Bohm particle at the exact line of symmetry intersecting what would be the intersection point is zero. In terms of the quantum potential, what happens is that it grows infinitely large at that point in configuration space, pushing all particles away. The result: Bohm trajectories bounce at I! Loosely put, a particle from A will "ride" that wavepacket until location I, but there, where the $x$-up and $x$-down wavepackets overlap, the particle will jump ship and hitch a ride with the originally downward wavepacket, creating the bounce. Particles found at B' originate at A and those found at A' originate at B, just the opposite of what is normally assumed. 


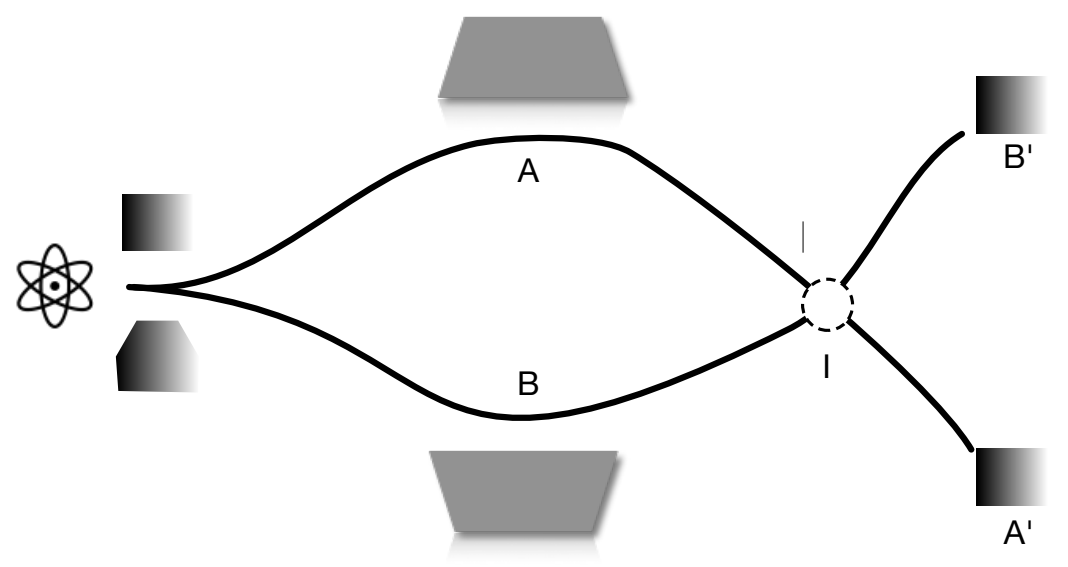

Fig. 3: Two Path Setup

You might reply that the standard textbook doesn't clearly commit to some ontology traveling from A to A' or B to B'. I agree that, ontologically speaking, the standard interpretation says little that is clear. Yet conventional quantum wisdom here is not ambiguous. In fact, the understanding that what is found at A' came from A is so strong that it was a central premise in an attempt by Englert et al 1992 to falsify Bohm's theory. In the so-called "surrealistic trajectories" debate, beautifully diagnosed by Barrett 2000, the main issue was that Englert et al assumed that they knew, from quantum theory, that the "real" trajectories didn't bounce.

\subsection{Bohr Orbits}

Perhaps I'm missing Cordero's point. Cordero suggests that there are layers of models that exist in quantum physics. The idea might be that many of these models are shared in common amongst quantum programs. Although not purely quantum, there is, for example, the Bohr model of the atom, and the BCS model of superconductors. There are also whole theories and interpretations of this "middle layer," such as semiclassical mechanics and the semiclassical interpretation. Again I find myself drawn to Cordero's position. His picture of physics as complex sets of models covering different regimes is much more realistic than that of many philosophers. One wouldn't be surprised if Everett, Collapse and Bohm, ever the enemies, end up holding hands in peace at the semiclassical level. Alas, I don't think that is so either. Let's agree to relax what we mean by quantum. Now we just want significant claims about the 
unobservable that aren't purely in the classical domain. That might not be enough to justify a realism about the quantum, but it would be a start.

Start with Bohr orbits. Consider hydrogen again and now its orbits. In the Bohm theory, when the electron is not at rest it orbits the $\mathrm{z}$-axis with constant speed and radius and is independent of mass. In the Bohr model, by contrast, the electron traces out orbits in the equatorial plane and the radius is a function of mass. The orbits are around different axes and one is a function of mass yet the other isn't. (See Fortin et al 2017 for this case and more.) They're different and it's hard to see how one approximates the other in any way. Again, at the measurement level, the Bohmian will be able to explain why the Bohr model worked as well as it did; but the reason isn't that Bohm particles travel the same orbits.

\subsection{Semiclassical Particle in a Box}

A field known as semiclassical physics (associated with the physicist Gutzwiller) develops and examines connections between classical orbits and quantum fluctuations. In this area, a system is understood to be "semiclassical" if the classical action is large compared to quantum $\hbar$. One finds semiclassical trajectories in this approach as one deals with chaos and other topics in dynamical systems theory. A tempting thought is that we'll find that as we approach the observable level, there will be agreement below the surface of the observable on the semiclassical trajectories. However, Matzin and Nurock 2008 show that semiclassical orbits differ dramatically from Bohmian orbits.

A simple example of the difference is the particle in a box. Using the typical wavefunction for such cases and assuming one-dimension and fixed energy, the Bohm particle will just sit still (unless you pull a wall away rapidly (Callender and Weingard 1998)). The semiclassical orbits, by contrast, give two classical orbits at each position in the box, one going in each direction, i.e., particles bouncing back and forth. Matzin and Nurock display other examples of divergence too, including (not surprisingly) hydrogen. Bohm's theory suggests a physical picture demonstrably at odds with the trajectories used in a semiclassical treatment.

\subsection{Summary}

Most of what we say about the quantum realm is "interpretation" dependent. The research programs described here portray radically different worlds from top to bottom, agreeing on little more than what is observable. I provided some examples but could multiply them easily, e.g., Bohmian Fermi-Dirac particles are not always repelled nor Bose-Einstein particles always attracted (Holland 1995, 310). I could also have used more examples from Collapse or Everett, so the response that these cases just show that Bohm is weird or unusual isn't sustainable. I cannot prove Cordero wrong. I have not gone through all of quantum mechanics and shown that there is nothing safe from the blight. Some models 
and systems may be safe. But these would be more like small disconnected islands of reprieve, not anything like a full quarantine zone.

\section{Reaction: There Can Only Be One?}

If I am right, there are at least three major research programs that each portray different worlds but that are compatible with the current empirical evidence. This situation poses a threat to the epistemic ambitions of the realist, someone who believes that mature successful theories are well-confirmed and approximately true. We can't say one program is well-confirmed and approximately true if we know that there are two others, equally well-confirmed, that contradict its hypotheses.

Before considering realist reactions, note that the situation isn't horribly dire. We hardly have guaranteed underdetermination by an indefinite number of theories. Three is a small number. We could apportion our degrees of belief over these three programs and not be at a complete loss when it comes to claims about the unobservable quantum world. ${ }^{3}$

Quantum underdetermination isn't, therefore, a disaster for realism. It is still disappointing. Can we do better? Ultimately there are two options, fight or flight. Realism could retreat by restricting its ambitions to claims that are interpretation neutral, as Hoefer (this volume) does. Quarantine works if the wall is placed around the observable level. Arguably, it also works in the potentially unobservable classical domain sector of quantum theory - although this is a tricky question and I have my doubts. Alternatively, we can fight this judgement by turning up the "scientific" dial. That is, we can use traditional realist features such as simplicity, unification, explanatoriness, and so on to decide which research program is best. We agreed that there are at least three options when the dial is set at "scientific." That might be too low a standard. None of the programs are cheap philosophical playthings, but that doesn't mean they are all equally well confirmed.

Let's briefly explore the more aggressive option of turning up the dial. I began the essay with a metaphor based on Greg Egan's clever and sophisticated book, Quarantine. I end with one based on a terribly acted and weakly plotted fantasy film, The Highlander (1986). In the film a group of (nearly) immortal warriors battle through history, dying only through decapitation. The last remaining will win the Prize. Warriors get stronger each time they kill one of their own. They know that, in the end, there can only be one. While I don't expect proponents of the different research programs to go away any time soon

\footnotetext{
${ }^{3}$ Could we repartition our programs and narrow down to two? What I have in mind is focusing on ontology and tipping theories into wavefunction-only and wavefunction-plus camps. Some versions of Everett and Collapse will then join Bohm in the wavefunction-plus camp, as they add a beable to the wavefunction in their basic ontology. I'm not a big fan of this repartitioning as it obscures major differences. For instance, Wallace 2017 shows that the branches corresponding to wavefunction-only GRW tails are qualitatively different from their Everettian counterparts, so the wavefunction-only camp includes very different worlds. More importantly, scientific realism is not only about the ontology but also the laws of the theory, and this partitioning ignores that fact.
} 
- and I certainly hope that they don't resort to Highlander-like tactics - it may be that when we turn up the dial, only one remains.

But which one?

Wallace (this volume) asserts that there is no underdetermination in quantum mechanics, that there is only Everett. His argument is that Everett and only Everett has been successfully applied to all of current physics. Bohm and Collapse lag behind, slogging their way through the history of quantum theory. Specifically, those research programs must develop relativistic and field theoretic versions of quantum theory, whereas it takes no time at all to make these versions Everettian. Put in terms of research programs, the idea is that the Everettian program is ahead of its rivals. When the dial is set to include empirical reach or size of domain, there is no underdetermination serious enough to cause alarm. ${ }^{4}$

Some proponents of structural realism also claim that there is no underdetermination; indeed, some motivate this type of realism via its ability to overcome quantum underdetermination. Since the mathematical structure of Collapse, Bohm and Everett differ so markedly, how could such a position ever get off the ground? In at least one case, the answer is that such realists deny that Collapse and Bohm merit consideration! Ladyman and Ross 2007, in their polemic against unmoored speculative metaphysics, claim that any response to the measurement problem that takes standard quantum mechanics to be an incomplete description of reality is an example of extravagant epistemically irresponsible metaphysics rather than good metaphysics or science (181). We can understand this as turning the dial to a very specific (and odd) setting. ${ }^{5}$

For a quite different judgement, consider Jean Bricmont's 2016 position when confronting quantum underdetermination. He argues that "there is no existing alternative to de Broglie-Bohm that reaches the level of clarity and explanatory

\footnotetext{
4 To be clear, the issue is more subtle than just described. There are indeed plenty of Bohmian field theories. There are also extensions of the theory to quantum gravity, quantum cosmology, superstring theory, quantum chemistry, and more. If Bohmian or Collapse answers to problems in new realms can't be reproduced by Everett then it's not clear who is more progressive. The Bohmian answer to the problem of time in canonical quantum gravity, for example, is not replicable in an Everettian framework, nor is the Collapse approach to the information loss paradox. The reason I put these points aside for the moment is that, overall, I agree that quantum field theory as a target dwarfs these examples in importance, and it's also fair to say that Bohmian or Collapse versions of the standard model of particle physics are a ways off.

5 I cannot fully respond to this charge here due to the editor's demand for polite, professional language. I'll just note that the following people have developed "completions" of quantum mechanics: Louise de Broglie, John Slater, Erwin Madelung, Albert Einstein, Nathan Rosen, Jean-Pierre Vigier, David Bohm, Hans Freistadt and John Bell. This list includes some of the top physicists who have ever lived. Scores of mathematicians and physicists the world over continue this work, e.g., Peter Holland, Sheldon Goldstein, Detleff Dürr, B.J. Hiley, Nino Zanghi, Roderich Tumulka, and publish rigorous advances in the best physics journals, e.g., Physical Review. Bohm's original paper has over 5000 citations. Appeal to authority is an improper argument form, I agree, but we can use it as a shortcut to a longer case I could make and ask: given the extrinsic markers of epistemic quality just listed, which is the more likely to be extravagant metaphysics, or a progressive research program, the work of Einstein, Bell and others in our best physics journals, or ontic structural realism?
} 
power of the latter" (228). Bricmont sees the threat of quantum underdetermination and adopts the Highlander move of eliminating alternatives. Unlike Wallace or Ladyman and Ross, he opts for Bohm as the last one standing, a judgement based on emphasizing explanatory virtues. We might understand Bricmont as employing confirmation understood as inference to the best explanation (Lipton 1991). According to such theories, for $T$ to be confirmed by $E, T$ must not only imply $E$ but $T$ must be the best explanation of $E$. Bohmians feel that the nuts-and-bolts accounts the theory provides of the stability of matter, uncertainty relations, interference, apparent wavefunction collapse, tunneling phenomena, and more is a major reason to adopt the theory. In a note added to the paperback edition of his Bohmian masterpiece, Holland stresses that the primary virtue of the Bohm program is its "quality of explanation" (1995, xix), much like that he finds in Darwinian reasoning. If this is right - and it certainly fits with my thoughts on the matter - one can imagine a Lipton-style argument to the effect that Bohm is better confirmed than its rivals. We turned up the dial once and eliminated the Cartesian demon. We turn the knob again and the same kinds of explanatory virtues leave only Bohm.

The debate between Wallace and Bricmont isn't likely to be settled anytime soon. The reason is that the very features that allow the Everettian interpretation its easy extension to new physics are precisely the same features that invite its problems and whose solutions by other programs lead to their explanatory virtues. Collapse swerves and Bohmian beables make probabilities relatively straightforward in these theories, for instance, whereas understanding probability is massively problematic in Everett. Moreover, it's these beables and swerves that allow the nuts and bolts explanatory narratives that attract Bohmians (and presumably, Collapse theorists). Yet it's these very swerves and beables that demand new physics to be developed when quantum theory is applied to new regimes, making extensions hardly automatic. The Everettian program is a "minimalist" one, little more than the quantum formalism itself coupled to a new rule for reinterpreting our definite empirical outcomes. So it is little wonder that it can be "successfully applied" to new physics. To someone engaged in a competing research program, trumpeting Everett's easy application to new physics sounds like a thief bragging about how little they had to work for their reward. After all, the Copenhagen interpretation - and Cartesian demon theory, for that matter - can make the same boast, yet many would agree that Copenhagen — and Cartesian demon theory — are so theoretically deficient as to take themselves out of the running. They earn their empirical progressiveness at the cost of providing a decent theory. Naturally, the Everettian sees matters precisely the other way around. Why are these "nuts and bolts" explanations, whatever they are, so great, and in particular, relevant to confirmation? Absent a compelling reply, and failing to see Everett as theoretically unsatisfactory, the choice for the empirically more progressive program is to them a no-brainer.

My own sympathies lie with the more "nuts-and-bolts" approaches. The point I want to make, however, is that we're pretty close to philosophical bedrock at this point. The Everettian and Bohmian described above aren't merely disagreeing on the correct dial setting, but they are disagreeing on the nature of 
the dial. Put somewhat simplistically, the Everettian uses a dial that represents size of empirical domain whereas the Bohmian uses a dial that represents explanatory virtues. The choice of research program therefore hangs on deep, hotly contested and familiar matters in philosophy of science-in particular, the relationship, if any, between explanatory virtues and confirmation.

In sum, we have serious scientific underdetermination. The nightmare of scientific realists is real. We're unlikely to secure any quarantine zone that retains much one can trust in the quantum realm. Because the differences between programs are so stark, it's tempting to go into Highlander mode and declare that there can be only one. But that debate - who remains standing won't go away soon because its resolution hangs on philosophical matters that are gridlocked. Welcome, scientific realists, to the quantum foundations. ${ }^{6}$

\section{References}

[1] Allori, V., Tumulka, R. and N. Zanghì, 2008. "On the Common Structure of Bohmian Mechanics and the Ghirardi-Rimini-Weber Theory", British Journal for the Philosophy of Science 59, 353-389.

[2] Allori, V., Tumulka, R. and N. Zanghì, 2011. "Many-Worlds and Schrödinger's First Quantum Theory", British Journal for the Philosophy of Science 62, 1-27.

[3] Barrett, J. A. 2000. "The Persistence of Memory: Surreal Trajectories in Bohm's Theory," Philosophy of Science 67(4), 680-703.

[4] Bell, J.S. 1987. Speakable and Unspeakable in Quantum Mechanics. Cambridge: Cambridge University Press.

[5] Bohm, D. 1952. "A Suggested Interpretation of the Quantum Theory in Terms of "Hidden" Variables. I. Physical Review, 85(2), 166.

[6] Bricmont, J. 2016. Making Sense of Quantum Mechanics. Springer

[7] Callender, C. 2015. "One World, One Beable" Synthese 192, 3153-3177.

[8] Callender, C. and Weingard, R. 1998. "Nonlocality in the Expanding Infinite Well" Foundations of Physics Letters 11, 495-98.

[9] Callender, C. and Weingard, R. 1994. "A Bohmian Model of Quantum Cosmology" Philosophy of Science 1994, 1, S228-237.

[10] Cordero, A. 2001. "Realism and Underdetermination: Some Clues from the Practices-up Philosophy of Science 3, S301-

\footnotetext{
${ }^{6}$ Many thanks to Adam Chin, John Dougherty, Carl Hoefer, Anncy Thresher and especially Kerry McKenzie, Chip Sebens and the referees for incredibly helpful feedback on a draft of this paper.
} 
[11] Daumer, M. Dürr, D. Goldstein, S. and N. Zanghì. 1996. "Naive Realism about Operators" Erkenntnis 45, 379-397.

[12] Dürr, D., S. Goldstein, and N. Zanghì. 1992. Quantum equilibrium and the origin of absolute uncertainty. Journal of Statistical Physics 67: 843-907.

[13] Englert, B.G., Scully, M. Sussman, G. and H. Walther, 1992. "Surrealistic Bohm Trajectories", Z. Naturforsch. 47a, 1175-1186.

[14] Everett, H. 1957. "The 'Relative State' Formulation of Quantum Mechanics", Review of Modern Physics, 29, 454-462.

[15] Fortin, S., Lombardi, O., Martınez Gonzalez, J.C. 2017. "The Relationship between Chemistry and Physics from the Perspective of Bohmian Mechanics" Found. Chem. 19, 43-59.

[16] Ghirardi, G.C., Rimini, A., and Weber, T. (1986). "Unified dynamics for microscopic and macroscopic systems". Physical Review D. 34: 470.

[17] Healey, R. 2017. "Quantum-Bayesian and Pragmatist Views of Quantum Theory", The Stanford Encyclopedia of Philosophy (Spring 2017 Edition), Edward N. Zalta (ed.), URL = $<$ https://plato.stanford.edu/archives/spr2017/entries/quantumbayesian $/>$.

[18] Holland, P. R. (1993/1995). The Quantum Theory of Motion. Cambridge: Cambridge University Press.

[19] Ladyman, J. and Ross, D. 2007. Every Thing Must Go: Metaphysics Naturalized. Oxford: Oxford University Press.

[20] Lakatos (1978). The Methodology of Scientific Research Programmes: Philosophical Papers Volume 1. Cambridge: Cambridge University Press

[21] Laudan, L. and Leplin, J., 1991, "Empirical Equivalence and Underdetermination", Journal of Philosophy 88, 449-472.

[22] Lipton, P. 1991. Inference to the Best Explanation. London: Routledge.

[23] Matzin, A. and Nurock, V. 2008. "Classical and Bohmian Trajectories in Semiclassical Systems: Mismatch in Dynamics, Mismatch in Reality?" Studies in History and Philosophy of Modern Physics 39, 17-40.

[24] Maudlin, T. forthcoming.

[25] McKenzie, K. 2017. "Ontic Structural Realism" In Philosophy Compass, April 2017

[26] Norsen, T. 2013. "The Pilot-wave Perspective on Quantum Scattering and Tunneling" American Journal of Physics 81, 258-266 
[27] Norton, J. 2008. "Must Evidence Underdetermine Theory?" in The Challenge of the Social and the Pressure of Practice: Science and Values Revisited, M. Carrier, D. Howard, and J. Kourany (eds.), Pittsburgh: University of Pittsburgh Press, 17-44.

[28] Okon, E. and Sudarsky, D. 2015. "The Black Hole Information Paradox and the Collapse of the Wave Function", Foundations of Physics 45, 461-470.

[29] Peruzzi, G., and Rimini, A. 2000. Compoundation invariance and Bohmian mechanics. Foundations of Physics 30: 1445-1472.

[30] Sebens, C. 2015a. "Quantum Mechanics as Classical Physics" Philosophy of Science 82, 266-291.

[31] Sebens, C. 2015b. "Killer Collapse: Empirically Probing the Philosophically Unsatisfactory Region of GRW" Synthese 8, 2599-2615.

[32] Skow, B. 2010. "On a Symmetry Argument for the Guidance Equation in Bohmian Mechanics", International Studies in the Philosophy of Science 24, 393-410.

[33] Struyve, W. 2011, "Pilot-wave Approaches to Quantum Field Theory", Journal of Physics: Conference Series 306, 012047.

[34] Wallace, D. 2014. Life and Death in the Tails of the GRW Wave Function. arXiv:1407.4746

[35] Wallace, D. 2018. Quantum theory as a framework, and its implications for the quantum measurement problem, this volume.

[36] Weingard R. 1996. "Exotic (Quixotic?) Applications of Bohm Theory". In: Clifton R. (eds) Perspectives on Quantum Reality. The University of Western Ontario Series in Philosophy of Science 57. Springer, Dordrecht. 